\title{
Right Breast
}

National Cancer Institute

\section{Source}

National Cancer Institute. Right Breast. NCI Thesaurus. Code C47856.

The hemispheric projection, including the mammary gland, located on the anterior portion of the chest, lateral to the midline, on the side of the body to the east when facing north. 